\title{
Ocorrência da coinfecção leishmaniose tegumentar americana/HIV no Estado do Maranhão
}

\author{
Occurrence of cutaneous leishmaniasis/HIV co-infection in the State of Maranhão \\ La aparición de la coinfección leishmaniasis cutánea/VIH en el Estado de Maranhão
}

Romário de Sousa Oliveira ${ }^{1 *}$, Karen Brayner Andrade Pimentel ${ }^{2}$, Francisco José Sousa Magalhães ${ }^{1}$, Glícia Cardoso Nascimento ${ }^{3}$, Lúcio Lauro Leite dos Santos ${ }^{4}$, Luciano André Assunção Barros ${ }^{1}$, Valéria Cristina Soares Pinheiro'.

\section{RESUMO}

Objetivo: Caracterizar a dinâmica temporal, espacial e os fatores clínico-epidemiológicos relacionados à ocorrência da coinfecção leishmaniose tegumentar americana/HIV no Maranhão. Métodos: Trata-se de um estudo ecológico com análise espacial e temporal dos casos de coinfecção leishmaniose tegumentar americana/HIV. Foram coletados os casos notificados no período de 2008 a 2017, segundo as variáveis sociodemográficas, clínicas e laboratoriais. Os dados foram tabulados utilizando-se o software Statistical Package Social Science versão 18. Para a distribuição espacial dos casos utilizou-se o software QGIS versão 2.14.9. Resultados: No período de 2008 a 2017, foram notificados 120 casos de coinfecção. O maior número ocorreu em indivíduos do sexo masculino $(76,7 \%)$, com 31 a 45 anos (33,3\%), que residiam em zona urbana (50,8\%) e tinham 1 a 4 anos de estudos (31,7\%). A forma clínica cutânea foi predominante (93,3\%). A droga leishmanicida mais utilizada foi o antimonial pentavalente $(89,2 \%)$. São Luís, Imperatriz, Centro do Guilherme e Açailândia foram os municípios com maiores registros de casos de coinfecção. Conclusão: A coinfecção leishmaniose tegumentar americana/HIV no Maranhão tem ocorrência heterogênea, com expressivo número de casos registrados. Tal fato reflete a importância do fortalecimento de medidas proativas no contexto da assistência à saúde.

Palavras-chave: Leishmaniose cutânea, Vírus da imunodeficiência humana, Doenças negligenciadas.

\begin{abstract}
Objective: To characterize the temporal and spatial dynamics and the clinical-epidemiological factors related to the coinfection occurrence of cutaneous leishmaniasis/HIV in the state of Maranhão. Methods: This is an ecological study with spatial and temporal analysis of coinfection cases of cutaneous leishmaniasis/HIV. The cases reported in the period from 2008 to 2017 were collected according to the sociodemographic, clinical and laboratory variables. The data were tabulated using the software Statistical Package Social Science version 18. For the spatial distribution of the cases, the software QGIS version 2.14 .9 was used. Results: In the period from 2008 to 2017, 120 cases of coinfection were reported. The highest number occurred in males (76.7\%), within 31 to 45 years old (33.3\%), who lived in urban areas (50.8\%) and had 1 to 4 years of schooling (31, $7 \%)$. The cutaneous clinical form was predominant (93.3\%). The most used leishmanicide drug was pentavalent antimonial (89.2\%). São Luís, Imperatriz, Centro de Guilherme and Açailândia were the municipalities with the highest number of coinfection cases. Conclusion: The cutaneous leishmaniasis/HIV coinfection in the state of Maranhão has a heterogeneous occurrence, with a significant number of registered cases. This fact reflects the importance of strengthening proactive measures in the context of health care.
\end{abstract}

Keywords: Cutaneous leishmaniasis, Human immunodeficiency virus, Neglected diseases.

${ }^{1}$ Universidade Estadual do Maranhão (UEMA), Caxias-Maranhão.

2 Universidade Federal do Maranhão (UFMA), São Luís-Maranhão.

${ }^{3}$ Universidade Federal do Piauí (UFPI), Teresina-Piauí.

${ }^{4}$ Universidade Estadual do Piauí (UESPI), Teresina-Piauí. *E-mail: rsobioq@hotmail.com

SUBMETIDO EM: 3/2019

ACEITO EM: 4/2019

PUBLICADO EM: 6/2019 


\section{RESUMEN}

Objetivo: Caracterizar la dinámica temporal, espacial y los factores clínico-epidemiológicos relacionados con la aparición de coinfección de leishmaniasis cutánea / VIH en el estado de Maranhão. Métodos: Se trata de un estudio ecológico con análisis espacial y temporal de los casos de coinfección de leishmaniasis cutánea / VIH. Los casos notificados en el período de 2008 a 2017 se recogieron de acuerdo con las variables sociodemográficas, clínicas y de laboratorio. Los datos se tabularon en el software Statistical Package Social Science versión 18. Se analizó la distribución espacial de los casos en el software QGIS versión 2.14.9. Resultados: En el período de 2008 a 2017, se notificaron 120 casos de coinfección. El mayor número se produjo en varones (76,7\%), entre 31 y 45 años (33,3\%), que vivían en áreas urbanas (50,8\%) y tenían de 1 a 4 años de escolaridad (31,7\%). La forma clínica cutánea fue predominante $(93,3 \%)$. El fármaco leishmanicida más utilizado fue el antimonial pentavalente (89,2\%). São Luís, Imperatriz, Centro de Guilherme y Açailândia fueron los municipios con el mayor número de casos de coinfección. Conclusión: La coinfección cutánea de leishmaniasis / VIH en el estado de Maranhão tiene una incidencia heterogénea, con un número significativo de casos registrados. Este hecho refleja la importancia de fortalecer las medidas proactivas en el contexto de la atención de salud.

Palabras clave: Leishmaniasis cutánea, Virus de la inmunodeficiencia humana, Enfermedades desatendidas-

\section{INTRODUÇÃO}

A leishmaniose tegumentar americana (LTA) é uma patologia tropical causada por protozoários pertencentes ao gênero Leishmania (MOHAMMAD A, et al., 2016). Em geral, sua transmissão ocorre através da picada de flebotomíneo fêmea infectada, embora seja possível a transmissão de humanos para humanos através do compartilhamento de agulhas. A doença apresenta diferentes padrões de transmissibilidade, fato que contribui para a sua ampla distribuição no mundo (VRIES JCM, et., 2015).

Nas Américas, a doença se caracteriza como um problema importante na saúde pública (ALVAR J, et al., 2012). No Brasil, a LTA apresenta importantes alterações no seu padrão de endemicidade em decorrência da sua expansão desordenada em áreas de desmatamento recente, o que potencializa a exposição ao vetor transmissor e o surgimento de novos focos (BRASIL, 2017). Em relação a epidemiologia da LTA no Brasil, verifica-se registros de casos autóctones em todos os estados brasileiros, sendo responsável por $70-75 \%$ da incidência de casos no mundo e com diferentes perfis epidemiológicos e padrões de transmissão, sujeitos a alterações socioambientais (MELCHIOR LAK, et al., 2017).

A infecção por LTA tem a possibilidade de assumir formas que podem determinar lesões destrutivas, desfigurantes e também incapacitantes, com grande repercussão no campo psicossocial do indivíduo em decorrências das lesões cutâneas e das deformidades resultantes da manifestação cutânea, fato agravado principalmente na vigência de imunodepressão (BENNIS I, et al., 2018; SCOTT P e NOVAIS FO, 2016).

A infecção por LTA possui apresentações clínicas que podem cursar com lesões cutâneas destrutivas, desfigurantes e tem a possibilidade de assumir deformidades resultantes da manifestação cutânea, fato agravado principalmente na vigência de imunodepressão, com grande repercussão no campo psicossocial do indivíduo (BENNIS I, et al., 2018; S COTT P e NOVAIS FO, 2016).

A LTA pode se disseminar de forma atípica nos pacientes imunossuprimidos, como no caso da infecção pelo human immunodeficiency virus (HIV) (GUERRA JA, et al., 2011). O HIV é um retrovírus de grande importância na saúde pública em decorrência da sua expansão e morbimortalidade associada principalmente à acquired immune deficiency syndrome (AIDS). A infecção pelo HIV em áreas endêmicas de LTA favorece a ocorrência de casos de coinfecção com grande impacto nos serviços de saúde (HEMELAAR J, et al., 2011).

$\mathrm{Na}$ última década, se tem verificado mudanças no perfil de coinfecção LTA/HIV, fato justificado, principalmente, pela existência de sobreposição espacial na ocorrência das duas doenças, com significativo impacto na sobrevida dos pacientes (TANGIE LN, et al., 2017). De maneira geral, se verificou um aumento no registro de casos de LTA/HIV nos últimos 20 anos, principalmente na região nordeste do Brasil (GOMES 
MLDS, et al., 2011). Entretanto, no Estado do Maranhão, pouco se conhece sobre a ocorrência e distribuição desta coinfecção. Nesse contexto, este estudo objetivou caracterizar a dinâmica temporal, espacial e os fatores clínico-epidemiológicos relacionados à ocorrência da coinfecção LTA/HIV no Maranhão, durante o período de 2008 a 2017, com intuito de gerar subsídios para a definição de medidas controle e prevenção no contexto da vigilância em saúde pública.

\section{MÉTODOS}

Trata-se de um estudo ecológico com análise temporal e espacial, através de levantamento de fontes secundárias referente aos casos autóctones da coinfecção LTA/HIV no Maranhão. Os dados foram obtidos na base de dados online do Sistema de Informação de Agravos de Notificação (SINAN). Foram selecionados os casos de LTA com coinfecção pelo HIV, notificados no período de 1ํ de janeiro de 2008 a 31 de dezembro de 2017. Os dados do Índice de Desenvolvimento Humano Municipal (IDHM), que avalia o desenvolvimento dos municípios brasileiros e as características internas de desigualdades presentes em cada região, foram obtidos através da plataforma online do Atlas do Desenvolvimento Humano no Brasil.

As variáveis analisadas foram sexo, faixa etária, raça/cor, escolaridade, zona de habitação, forma clínica, dados laboratoriais (parasitológico direto, Intradermorreação de Montenegro, histopatológico), tratamento (droga inicial administrada), critério de confirmação (laboratorial ou clínico epidemiológico). Os dados obtidos foram tabulados utilizando-se o software Statistical Package Social Science (SPSS) versão 18.0 para Windows. Para a elaboração dos mapas temáticos foi utilizado o software QGIS versão 2.14.9. O representativo de base cartográfica adotado foi o Sistema de Referência Geocêntrico para as Américas 2000. Em relação aos aspectos éticos, não foi necessário a submissão do presente estudo ao Comitê de Ética em Pesquisa, já que são dados secundários, de livre acesso, provenientes do Sistema Nacional de Agravos de Notificação. No entanto, foram respeitadas as questões éticas norteadas pela Resolução 466/2012 do Conselho Nacional de Saúde.

\section{RESULTADOS}

No período de 2008 a 2017 foram notificados 120 casos de coinfecção LTA/HIV no Maranhão, com uma média de 12 casos por ano. O maior e o menor número de notificações foram registrados nos anos de 2015, com 20 (16,6\%), e em 2009, com 4 (3,3\%) casos, respectivamente (Figura 1).

Figura 1 - Distribuição temporal dos casos de coinfecção LTA/HIV no Estado do Maranhão, 2008-2017.

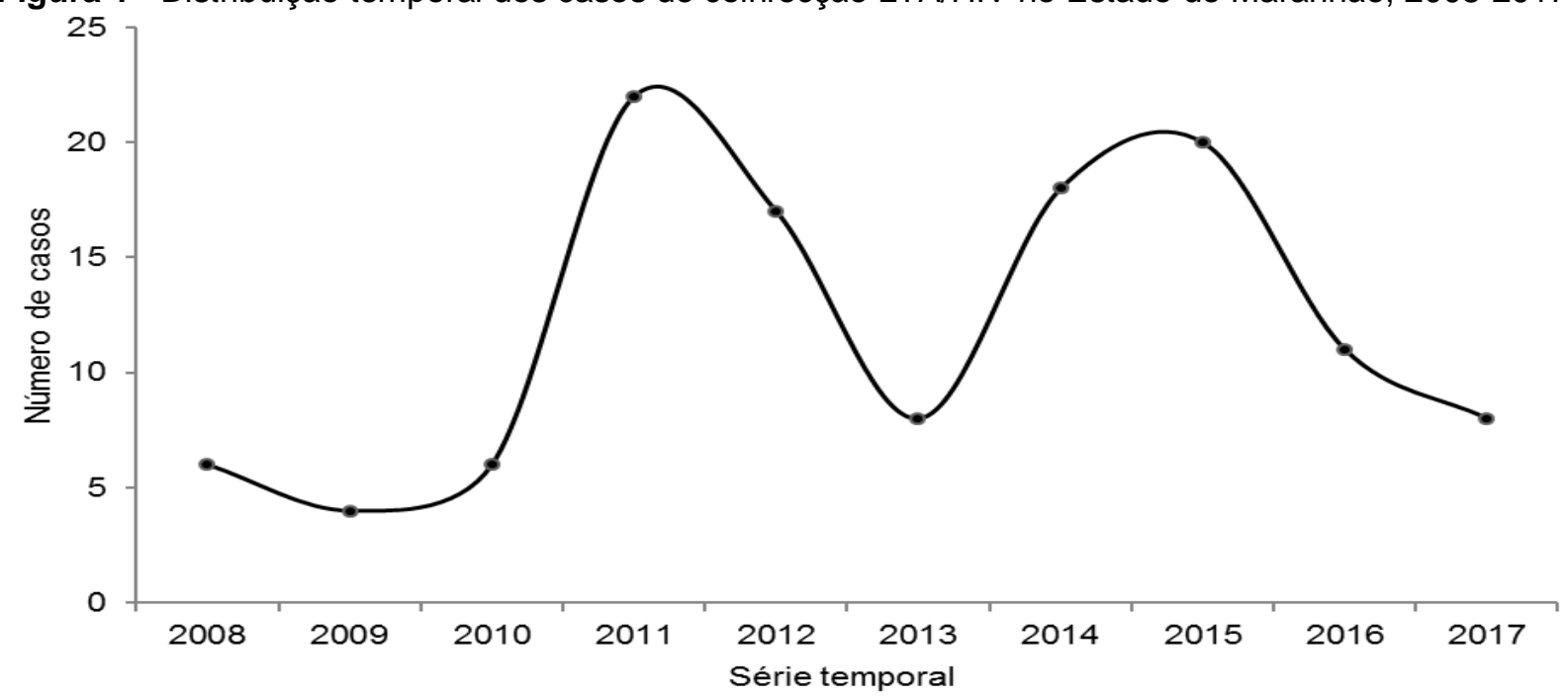

Fonte: Sistema de Informação de Agravos de Notificação - Sinan, 2018. 
A Tabela 1 analisa as variáveis demográficas relativas aos casos de coinfecção LTA/HIV. Os indivíduos do sexo masculino foram os mais acometidos (76,7\%). Em relação a faixa etária, verificou-se maior ocorrência entre indivíduos de 31 a 45 anos (33,3\%). A cor parda foi predominante $(75 \%)$. A baixa escolaridade foi um fator relevante, já que em $31,7 \%$ dos casos, os indivíduos tinham até 4 anos de estudo e $6,7 \%$ eram analfabetos. Do total de casos identificados, $50,8 \%$ residiam em zona urbana, no entanto, é expressivo o número de notificações em indivíduos residentes em zona rural $(47,5 \%)$.

Tabela 1 - Características sociodemográficas dos casos de coinfecção LTA/HIV no Estado do Maranhão, 2008-2017.

\begin{tabular}{|c|c|c|}
\hline Variáveis & $\mathbf{N}$ & $\%$ \\
\hline \multicolumn{3}{|l|}{ Sexo } \\
\hline Masculino & 92 & 76,7 \\
\hline Feminino & 28 & 23,3 \\
\hline \multicolumn{3}{|l|}{ Faixa etária } \\
\hline$<15$ anos & 17 & 14,2 \\
\hline 16 a 30 anos & 33 & 27,5 \\
\hline 31 a 45 anos & 40 & 33,3 \\
\hline 46 a 60 anos & 21 & 17,5 \\
\hline$>60$ anos & 9 & 7,5 \\
\hline \multicolumn{3}{|l|}{ Raça/cor } \\
\hline Branca & 13 & 10,8 \\
\hline Preta & 9 & 7,5 \\
\hline Amarela & 3 & 2,5 \\
\hline Parda & 90 & 75 \\
\hline Indígena & 3 & 2,5 \\
\hline Ignorado & 2 & 1,7 \\
\hline \multicolumn{3}{|l|}{ Escolaridade } \\
\hline Analfabeto & 8 & 6,7 \\
\hline 1 a 4 anos de estudo & 38 & 31,7 \\
\hline 5 a 8 anos de estudo & 23 & 19,2 \\
\hline 9 a 11 anos de estudo & 16 & 13,3 \\
\hline 12 anos ou mais & 1 & 0,8 \\
\hline Não se aplica & 6 & 5 \\
\hline Ignorado & 28 & 23,3 \\
\hline \multicolumn{3}{|l|}{ Zona de habitação } \\
\hline Urbana & 61 & 50,8 \\
\hline Rural & 57 & 47,5 \\
\hline Ignorado & 2 & 1,7 \\
\hline Total & 120 & 100,0 \\
\hline
\end{tabular}

Fonte: Sistema de Informação de Agravos de Notificação - Sinan, 2018. 
Na Tabela 2, observa-se as características clínicas e laboratoriais das notificações de coinfecção LTA/HIV. Houve predominância da forma cutânea $(93,3 \%)$. Em $74,2 \%$ dos casos notificados, o exame parasitológico foi positivo. A intradermorreação de Montenegro (IRM) não foi realizado em $62,5 \%$ dos casos. Em $66,7 \%$ dos casos não foi realizado a histopatologia. Em relação ao tipo de medicamento inicialmente administrado, o antimonial pentavalente foi o mais utilizado $(89,2 \%)$.

Tabela 2 - Características clínicas e laboratoriais dos casos de coinfecção LTA/HIV no Estado do Maranhão, 2008-2017.

\begin{tabular}{|c|c|c|}
\hline Variáveis & $\mathbf{n}$ & $\%$ \\
\hline \multicolumn{3}{|l|}{ Forma clínica } \\
\hline Cutânea & 112 & 93,3 \\
\hline Mucosa & 8 & 6,7 \\
\hline \multicolumn{3}{|l|}{ Parasitológico direto } \\
\hline Positivo & 89 & 74,2 \\
\hline Negativo & 8 & 6,6 \\
\hline Não realizado & 23 & 19,2 \\
\hline \multicolumn{3}{|c|}{ Intradermorreação de Montenegro } \\
\hline Positivo & 37 & 30,8 \\
\hline Negativo & 8 & 6,7 \\
\hline Não realizado & 75 & 62,5 \\
\hline \multicolumn{3}{|l|}{ Histológico } \\
\hline Encontro do parasita & 38 & 31,7 \\
\hline Compatível & 2 & 1,6 \\
\hline Não realizado & 80 & 66,7 \\
\hline \multicolumn{3}{|l|}{ Droga inicial administrada } \\
\hline Antimonial pentavalente & 107 & 89,2 \\
\hline Anfotericina B & 5 & 4,2 \\
\hline Outras & 2 & 1,6 \\
\hline Não utilizada & 1 & 0,8 \\
\hline Ignorado & 5 & 4,2 \\
\hline \multicolumn{3}{|l|}{ Critério de confirmação } \\
\hline Laboratorial & 107 & 89,2 \\
\hline Clínico epidemiológico & 13 & 10,8 \\
\hline Total & 120 & 100,0 \\
\hline
\end{tabular}

Fonte: Sistema de Informação de Agravos de Notificação - Sinan, 2018.

Para a análise da relação entre o IDHM e casos de coinfecção LTA/HIV no Maranhão, foram construídos mapas temáticos com sobreposição entre estas variáveis em dois intervalos temporais (2008 a 2012 e 2013 a 2017). O IDHM no estado do Maranhão apresenta grande heterogeneidade, com predominância de municípios com baixo e médio IDHM. Foi verificado aumento no número de municípios com baixo ou médio IDHM que notificaram casos novos da coinfecção, com destaque para Barra do Corda (1caso; IDHM 0,606), Bela Vista do Maranhão (1 caso; IDHM 0,554), Peritoró ( 1 caso; IDHM 0,564), Rosário ( 1 caso; IDHM 0,632) (Figura 2).

Em Centro do Guilherme, oeste maranhense, notificou o maior número de casos de coinfecção por LTA/HIV no período de 2008 a 2012 (24,4\%). Os municípios de Açailândia, São Luís e Imperatriz mostraram aumento do número de notificações dos casos de LTA/HIV. Entre o período de 2008-2012 foram registrados nesses municípios $2(1,7 \%), 3(5,5 \%)$ e $5(9 \%)$ casos respectivamente. Já entre os anos de 2013 a 2017 esse número foi de $5(7,6 \%), 5(7,6 \%)$ e $7(10,7 \%)$, respectivamente. 
Figura 2 - Mapa temático da distribuição do índice de desenvolvimento humano por município e sobreposição dos casos de coinfecção LTA/HIV no Estado do Maranhão, no período de 2008 a 2017.
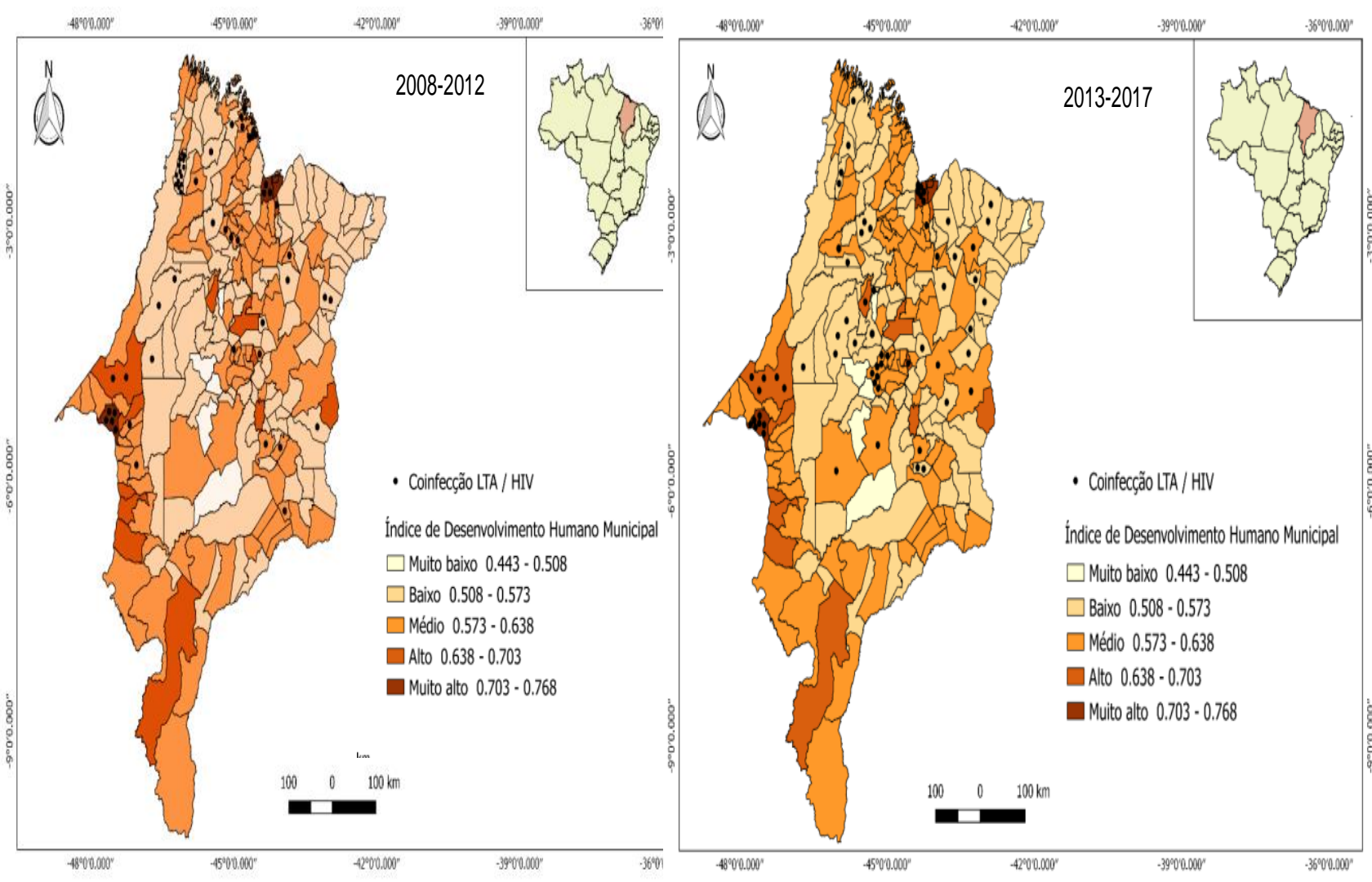

Fonte: Sistema de Informação de Agravos de Notificação - Sinan, 2018.

\section{DISCUSSÃO}

A ocorrência de coinfecção leishmania/HIV já foi registrada em cerca de 35 países e representa um desafio atual de saúde pública (LINDOSO JAL, et al., 2016). No Brasil, poucos são os relatos que descrevem a coinfecção LTA/HIV, principalmente no nordeste brasileiro (CAVALIN, RF, 2018). Este estudo demonstra que a ocorrência de casos de LTA/HIV no Maranhão apresenta disseminação heterogênea, com importante notificação de casos novos em municípios com IDHM baixo ou médio.

$\mathrm{Na}$ atualidade, tanto a LTA como o HIV são doenças de notificação compulsória. Dada a sua ocorrência e dispersão nos diversos estados brasileiros, existe na ficha de notificação um campo exclusivo para registro dos casos de coinfecção com HIV (BRASIL, 2017). A LTA não se encontra no rol principal de doenças oportunistas associadas ao HIV (DIRO E, et al., 2015) No entanto, é importante relatar que o acesso expandido aos antirretrovirais proporcionou aumento da expectativa de vida dos pacientes, alterando a tendência de mortalidade associada e mudanças no seu padrão epidemiológico, o que proporciona ampliação das zonas de sobreposição das duas doenças (SAAG MS, et al., 2018).

No período de 2008 a 2017, foram notificados 19791 casos de LTA no Maranhão, desde 0,83\% correspondiam a registros de coinfecção com HIV. Foi verificado maior ocorrência em indivíduos do sexo masculino, o que corrobora as informações de estudo realizado na Amazônia brasileira que revelou uma predominância de casos em indivíduos adultos jovens do sexo masculino (GUERRA JA, et al., 2011). Estudos afirmam que o fator etário contribui para a associação desta parasitose com o HIV, já que este ainda afeta de forma expressiva indivíduos nessa faixa etária (GRIENSVEN JV, et al., 2014; ARONSON N, et al., 2016). 
Neste estudo, a forma clínica mais frequente foi a cutânea. Esse resultado é semelhante ao encontrado em outros estudos masculino (GUERRA JA, et al., 2011). Na vigência da coinfecção LTA/HIV, o paciente pode apresentar quadro clínico atípico. Estudos apontam maior frequência de manifestação cutânea disseminada, incluindo relatos de visceralização da doença (MARTíNEZ DY, et al., 2018).

O tratamento da LTA na vigência de coinfecção com o HIV representa um desafio mundial de saúde pública (SILVA FC, et al., 2018). A terapêutica antileishmania tem como objetivo principal prevenir a morbidade da doença e evitar evoluções para formas desfigurantes (GALVÃO EL, et al., 2019). No Brasil, o medicamento de primeira escolha para os casos de coinfecção é o desoxilato de anfotericina B. Neste estudo, o tratamento foi realizado majoritariamente com o antimonial pentavalente, fato desaconselhado pelo Ministério da Saúde devido à grande toxicidade associada. É importante ressaltar que o seguimento durante e após o tratamento deve ocorrer por tempo indeterminando, com intuito de detectar recaídas e diminuir a morbimortalidade da doença. (BRASIL, 2017).

No contexto brasileiro, o estado do Maranhão apresenta um dos maiores índices de pobreza. Tal fato tem grande impacto na perpetuação de doenças infecciosas e parasitárias. Assim, redução das desigualdades relacionadas ao desenvolvimento humano deve ser entendido como uma prioridade das políticas públicas (CHOKSHI DA, 2018). É válido registrar a dificuldade de acesso aos serviços de saúde nos municípios interioranos, onde uma grande parcela da população reside em zona rural ou periurbana, o que representa um desafio na identificação, registro, conduta e seguimento desses casos e requer atenção dos serviços de atenção em saúde (BURNETT CFL, et al., 2016; KESSLER, M, et al, 2015).

\section{CONCLUSÃO}

Neste estudo, verificou-se que a coinfecção LTA/HIV foi registrada predominantemente em indivíduos do sexo masculino, adultos com nenhuma ou pouca escolaridade. Houve predominância da forma cutânea e o antimonial pentavalente foi o medicamento mais utilizado. A distribuição espacial dos casos foi heterogênea, predominando nos municípios de São Luís, Imperatriz, Centro do Guilherme e Açailândia. O Maranhão é um estado que requer atenção na dinâmica de ocorrência da coinfecção LTA/HIV, devido ao registro expressivo de casos dessas duas moléstias infecciosas.

\section{AGRADECIMENTOS}

Ao Conselho Nacional de Desenvolvimento Científico e Tecnológico (CNPq) pela concessão de bolsa de iniciação científica n 121824/2018-7.

\section{REFERÊNCIAS}

1. ALVAR J, et al. Leishmaniasis worldwide and global estimates of its incidence. PLoS One, 2012; 7(5): e35671.

2. ARONSON N, et al. Diagnosis and treatment of leishmaniasis: clinical practice guidelines by the Infectious Diseases Society of America (IDSA) and the American Society of Tropical Medicine and Hygiene (ASTMH). Clinical infectious diseases, 2016; 63(12): e202-e264.

3. BURNETT, CFL et al. Planejamento e gestão de cidades no Maranhão: o executivo municipal e o controle do solo urbano. São Luís: EDUEMA, 2016; 288p.

4. BENNIS I, et al. Psychosocial burden of localized cutaneous Leishmaniasis: a scoping review. BMC Public Health, 2018; 18:358.

5. BRASIL. Ministério da Saúde. Secretaria de Vigilância em Saúde. Departamento de Vigilância das Doenças Transmissíveis. Manual de Vigilância da Leishmaniose Tegumentar Americana. Brasília: Ministério da Saúde, 2017; 189p.

6. CAVALIN, RF. Coinfecção TB-HIV: análise espacial e temporal no município de São Paulo. Dissertação (Mestrado em Saúde Pública) - Faculdade de Saúde Pública, Universidade de São Paulo, São Paulo, 2018; 128 p.

7. CHOKSHI DA. Income, Poverty, and Health Inequality. Jama, 2018; 319(13): 1312-1313. 
8. DIRO E, et al. Atypical manifestations of visceral leishmaniasis in patients with HIV in north Ethiopia: a gap in guidelines for the management of opportunistic infections in resource poor settings. The Lancet Infectious Diseases, 2015; 15(1): 122-129.

9. GALVÃO EL, et al. How cutaneous leishmaniasis and treatment impacts in the patients' lives: A cross-sectional study. PloS one, 2019; 14(1): e0211374.

10. GOMES MLDS, et al. Coinfecção Leishmania-HIV no Brasil: aspectos epidemiológicos, clínicos e laboratoriais. Epidemiol Serv Saúde, 2011; 20(4): 519-526.

11. GRIENSVEN JV, et al. Leishmaniasis in immunosuppressed individuals. Clinical Microbiology and Infection, 2014; 20(4): 286-299.

12. GUERRA JA, et al. American tegumentary leishmaniasis and HIV-AIDS association in a tertiary care center in the Brazilian Amazon. Am J Trop Med Hyg, 2011; 85(3): 524-527.

13. HEMELAAR J, et al. Global trends in molecular epidemiology of HIV-1 during 2000-2007. AIDS (London, England), 2011; 25(5): 679- 89.

14. IBGE. Instituto Brasileiro de Geografia e Estatística. Censo 2010 [online]. Disponível em: http://www.ibge.gov.br/estado. Acesso em: 15 dez. 2018.

15. KESSLER, M, et al. Cobertura das estratégias de fortalecimento da atenção básica em saúde. Rev. pesqui. cuid. fundam. (Online), 2015; 7(3): 3050-3062.

16. LINDOSO JAL, et al. Leishmaniasis-HIV coinfection: current challenges. HIV AIDS (Auckl), 2016; 8:147-156.

17. MARTÍNEZ DY, et al. Tegumentary leishmaniasis and coinfections other than HIV. PLoS neglected tropical diseases, 2018; 12(3): e0006125.

18. MELCHIOR LAK, et al. Spatial and temporal distribution of American cutaneous leishmaniasis in Acre state, Brazil. Infect Dis Poverty, 2017; 6(1): 99.

19. MOHAMMAD A, et al. A Historical Overview of the Classification, Evolution, and Dispersion of Leishmania Parasites and Sandflies. PLOS Neglected Tropical Diseases, 2016; 10(6): e0004770.

20. SAAG MS, et al. Antiretroviral drugs for treatment and prevention of HIV infection in adults: 2018 recommendations of the International Antiviral Society-USA Panel. JAMA, 2018; 320(4): 379-396.

21. SCOTT $P$, NOVAIS FO. Cutaneous leishmaniasis: immune responses in protection and pathogenesis. Nature Reviews Immunology, 2016; 16(9): 581-592.

22. SILVA FC, et al. The Binomial Parasite-Host Immunity in the Healing Process and in Reactivation of Human Tegumentary Leishmaniasis. Frontiers in microbiology, 2018; 9(1308): 1-17.

23. TANGIE LN, et al. Cutaneous leishmaniasis in a severely immunocompromised HIV patient in Kumbo, Northwest region of Cameroon: case report. BMC Research Note, 2017; 10(1): 425.

24. VRIES JCH, et al. Cutaneous Leishmaniasis: Recent Developments in Diagnosis and Management. Am J Clin Dermatol, 2015; 16(2): 99-109. 